\title{
Overview of Normal Hematopoiesis
}

Mahmoud Gaballa and Carlos A. Ramos

\section{INTRODUCTION}

The formed elements of blood include various types of cells or cell fragments, each with different morphology and function. These elements are produced via a process known as hematopoiesis, during which hematopoietic stem cells (HSCs) proliferate and undergo self-renewal or differentiation into lineage-committed progenitors, which continue to differentiate into mature blood cells. This ability to self-renew and the ability to differentiate are two key characteristics of HSCs necessary for normal hematopoiesis $(1,2)$. Selfrenewal is the process by which stem cells enter the cell cycle to divide and give rise to more stem cells, thus preserving the stem cell pool. On the other hand, differentiation allows HSCs to develop into more mature cells with progressive lineage commitment. In general, the ability to self-renew diminishes as maturation and lineage commitment progresses.

The capacity of HSCs to differentiate into multiple cell lines is termed multipotency. The hematopoietic hierarchy is a well-orchestrated process that starts with HSCs developing into myeloid and lymphoid progenitor cells. Myeloid progenitor cells continue to develop into erythrocytes, platelets (through fragmentation of megakaryocytes), neutrophils, monocytes, basophils, and eosinophils. In contrast, lymphoid progenitor cells give rise to B lymphocytes, $T$ lymphocytes, natural killer (NK) cells, and a population of dendritic cells (Figure 1.1). Thus, a very complex process in the bone marrow gives rise to at least 10 cellular elements on a daily basis. Blood cell numbers are sustained within relatively narrow ranges through this process, with the ability to boost production if needed in states of increased demand. In this chapter, we illustrate our current understanding of hematopoiesis, its stages, the cellular and non-cellular elements involved, and its regulation. 


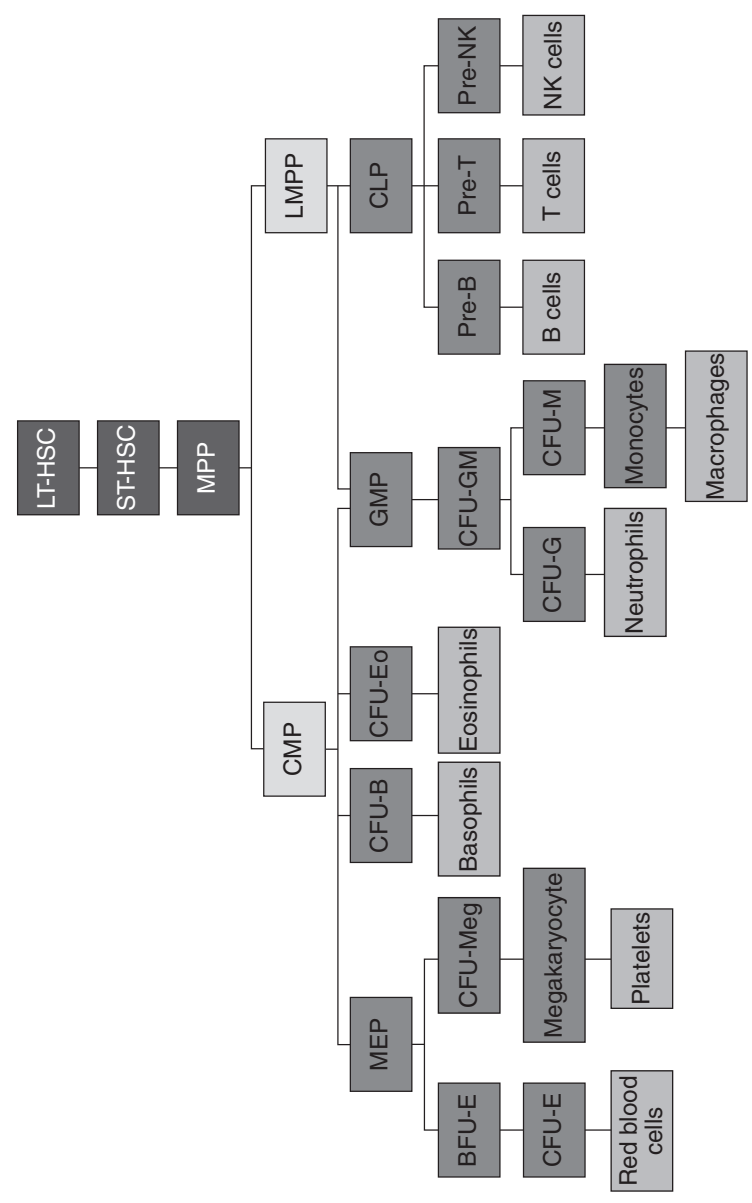

$\stackrel{\text { ह }}{\frac{1}{\varepsilon}}$

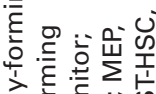

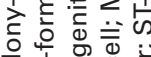

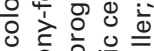

응 응 产

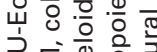

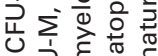

के मे है

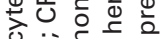

일

ᄃ

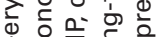

.

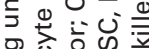

잉유

을 을 똔

눈 준

4흔은

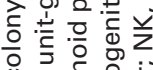

엉 둥 山े. 을 을

गे है

넌유웡

눌을

등응 응 흘 능

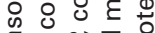

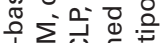

产 仓

ร

인 항믐

'ิ

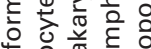

4 음

टे 응 듄

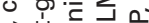

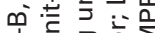

. ذई ฮ

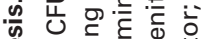

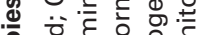

ํㅡㅇ 은 눈 눈응

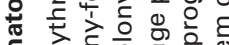

ह 중응

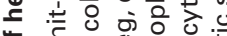

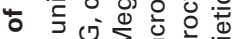

¿

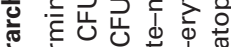

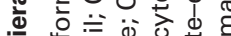

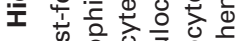

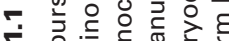

의

ب

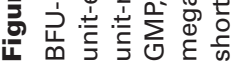




\section{STAGES AND SITES OF HEMATOPOIESIS}

Hematopoiesis starts during embryonic life and continues through fetal, neonatal, and adult life. Embryonic hematopoiesis comprises two waves of events. The first wave, often referred to as primitive hematopoiesis, takes place specifically in the extraembryonic yolk sac, where nucleated primitive erythrocytes are produced. This is a critical step for the development and survival of the embryo since those primitive erythrocytes are the first elements to facilitate oxygen transportation. In addition, myeloid cells produced in the yolk sac subsequently relocate to the central nervous system and skin to develop into microglia and Langerhans cells, respectively (3). In humans, primitive hematopoiesis lasts from day 19 until week 8 postconception. The second wave, characterized by intraembryonic hematopoiesis, takes place initially in the dorsal aorta (aortagonad-mesonephros or AGM region), where hematopoietic progenitors are produced from mesoderm derivatives (4). This process happens once in a lifetime and those cells later continue to develop to form adult HSCs, with the expression of major histocompatibility complex (MHC) class II and cluster of differentiation (CD)45.

In the fetus, hematopoiesis transitions first from the embryonic sites to the liver. At approximately 5 gestational weeks, the HSCs and progenitors travel from the yolk sac or AGM to the liver to establish hematopoiesis (5). At this stage of life, the liver serves as a vital hematopoietic organ where differentiation and expansion of HSCs take place, a process commonly referred to as definitive erythropoiesis (5). In addition, the placenta also becomes a major contributor to hematopoiesis, starting in fetal life and continuing until birth. Furthermore, some HSCs and progenitor cells leave the liver during fetal life and migrate to the spleen, where further differentiation into myeloid and lymphoid lineages occurs transiently (3).

At 4 to 5 months of gestation, hematopoiesis begins to occur in the bone marrow, a process termed medullary hematopoiesis, which continues after birth and throughout postnatal life. As we advance into adulthood, hematopoiesis slowly becomes confined to the skull, pelvic bones, vertebrae, and the metaphyseal region of long bones, with the diaphysis of long bones being gradually replaced with adipose tissue. Thus, the active bone marrow of children is proportionally much larger than that of adults, which is thought to occur due to the higher demand for red cells in neonatal and childhood life compared to adulthood. Importantly, in states of medullary insufficiency, as in patients with thalassemia and myelofibrosis, hematopoiesis can revert to its original sites, including the liver and spleen. This is termed extramedullary hematopoiesis. Figure 1.2 illustrates the sites of hematopoiesis in different stages of life. 


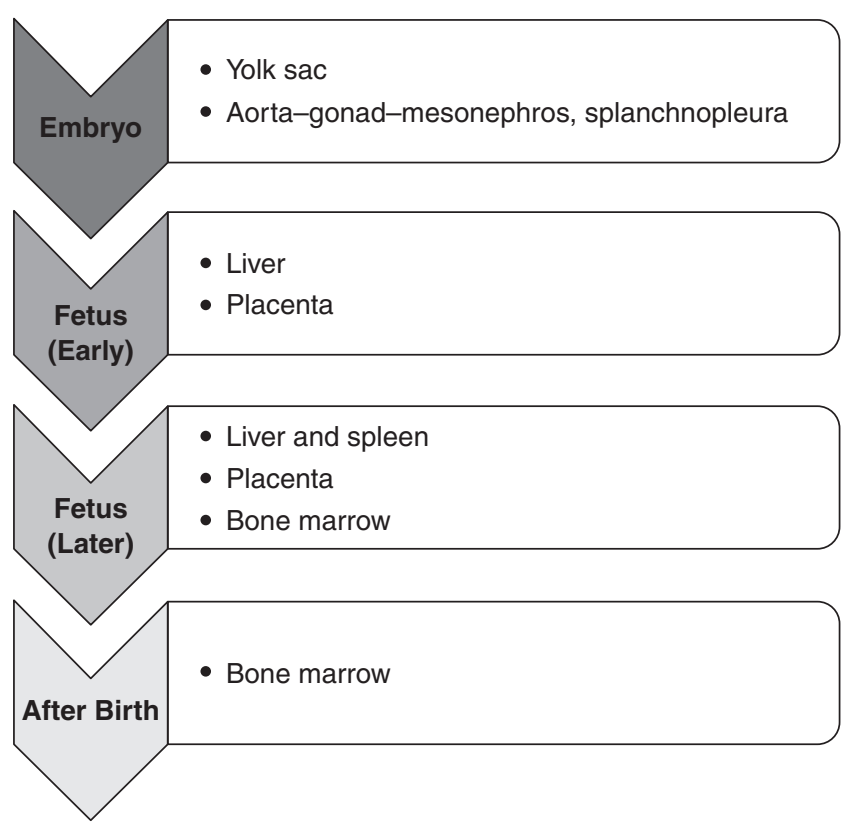

Figure 1.2 Sites of hematopoiesis in different stages of life.

\section{HSCs AND THE HEMATOPOIETIC HIERARCHY}

The hematopoietic differentiation cascade is akin to an inverted tree, starting with a small pool of HSCs that develop into a larger pool of progenitors, which then further differentiate into many intermediates and finally develop into different mature blood cells. As the cells travel down their hierarchy, they lose the ability to differentiate into other cell lines and progressively become committed to one lineage, that is, lineage-committed progenitors.

HSCs choose early whether to self-renew or to differentiate into more mature cells, with two models proposed to describe this choice, the stochastic (supportive) and the instructive. In the stochastic model, the choice of self-renewal versus differentiation is random. In contrast, in the instructive model, certain microenvironment factors and cytokines directly influence this decision. Notably, cytokines have critical but different roles in both models. In the instructive model, cytokines actively influence the decision, while in the stochastic model, cytokines do not influence the decision but support the survival and proliferation of progenitor cells that eventually differentiate into mature cells (6). This issue remains controversial with conflicting data supporting both models. 
HSCs are further subclassified as long term (LT-HSCs) and short term (ST-HSCs). The main role of LT-HSCs is self-renewal, and most of their progeny will follow this pathway, while lesser numbers become ST-HSCs (1). ST-HSCs have lower self-renewal potential and their predominant fate is to differentiate into multipotent progenitors (MPPs). These, in turn, give rise to common myeloid progenitors (CMPs) and lymphoid primed multipotent progenitors (LMPPs). CMPs and LMPPs then continue their differentiation into lineage-committed progenitors, ending with the different mature blood cells (1).

Hematopoietic progenitors can be classified into multilineage progenitors and single-lineage progenitors according to their differentiation potential. As illustrated in Figure 1.1, there are two main multilineage progenitors recognized, namely, the CMP and the common lymphoid progenitor (CLP; the immediate progeny of LMPPs). The CMPs give rise to erythrocytes, platelets (via megakaryocytes), basophils, eosinophils, neutrophils, and macrophages after a series of intermediate forms, which are generally named burst-forming units (BFUs) or colony-forming units (CFUs) (7). This nomenclature is derived from in vitro assays performed by culturing cells on methylcellulose, a semi-solid medium, mixed with different cytokines intended to induce differentiation into various lineages (8). The timing of appearance, the morphology, the cellular components, and the cytokine requirements of the colonies arising in these assays define each of the intermediate forms, with the convention that a cell population identified by a particular assay is referred to by the assay name. CLPs ultimately differentiate into $B$, $\mathrm{T}$, and NK cells. While myeloid and lymphoid lineages are thought to arise independently, some studies have suggested that the granulocyte-macrophage progenitor (GMP) can also be derived from LMP.

Whereas many of the intermediates are not morphologically distinguishable, the majority of the final differentiation steps toward individual lineages can be identified by conventional histochemistry, where a blast form gives rise to more mature forms that progressively resemble the fully differentiated elements. Table 1.1 describes the nomenclature of the different processes leading to production of mature blood cells. In addition, Table 1.2 lists the different types of mature blood cells as well as their morphologic characteristics and function.

\section{CLUSTERS OF DIFFERENTIATION}

As HSCs and progenitors differentiate, they acquire distinct cell surface antigen markers, also known as CDs, while losing the antigens associated with more primitive cells. CDs can be used 
Table 1.1 Hematopoietic Processes

\begin{tabular}{|c|c|c|}
\hline Process & Progenitor & Outcome \\
\hline Myelopoiesis & CMP & $\begin{array}{l}\text { Production of myeloid cells, which can } \\
\text { be granulocytic and non-granulocytic } \\
\text { - Granulocytic } \\
\text { - Neutrophils } \\
\text { - Eosinophils } \\
\text { - Basophils } \\
\text { - Non-granulocytic } \\
\text { - Monocytes and macrophages }\end{array}$ \\
\hline Lymphopoiesis & CLP & Production of B and T Iymphocytes \\
\hline Erythropoiesis & MEP & Production of red blood cells \\
\hline Thrombopoiesis & MEP & Production of platelets \\
\hline
\end{tabular}

to identify stem cells and diverse types of progenitors, intermediates, and mature blood cells of different lineages, even at stages when morphology alone would not be enough for cell identification. Identifying cell subpopulations via CDs is often done through monoclonal antibodies and flow cytometry, with marker panels being readily available commercially. The number of described CDs continues to grow and has exceeded 370 to date. Utilization of CDs in the clinical arena has revolutionized the way we understand, diagnose, classify, and treat hematologic diseases, and is now the standard of care to be incorporated in their diagnostic workup.

CDs are considered differentiation antigens, some of which are summarized in Table 1.3 (9), with many being membrane proteins that play an important role in hematopoiesis. These proteins often act as receptors or ligands with subsequently activated signal transduction pathways regulating the behavior of the cells expressing them. Other CDs are not involved in signal transduction, but have other functions such as mediating cell adhesion. Figure 1.3 illustrates selected examples of markers that are associated with different lineages.

CD34 and CD117 (c-KIT) are markers expressed by HSCs from the fetal stages of hematopoiesis through adult life. Importantly, HSCs and primitive progenitors lack CD38 (10). HSCs are rare, and thus, techniques that enhance their enrichment often rely on the identification of those surface markers and exclusion of antigens that define downstream lineages (so-called lineage negativity, Lin-) (11). One important clinical example is the use of CD34 to enrich hematopoietic grafts for HSCs. 


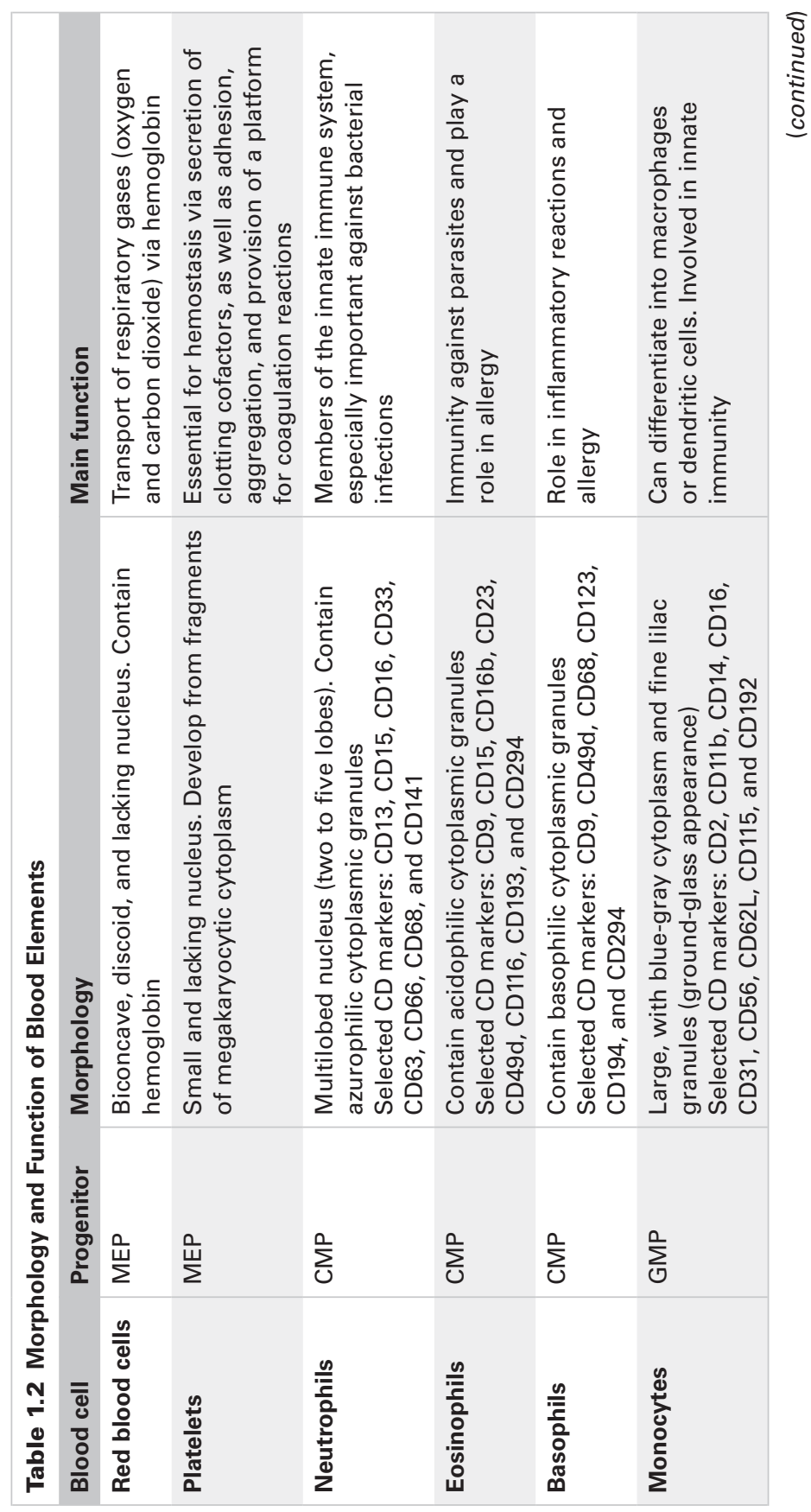




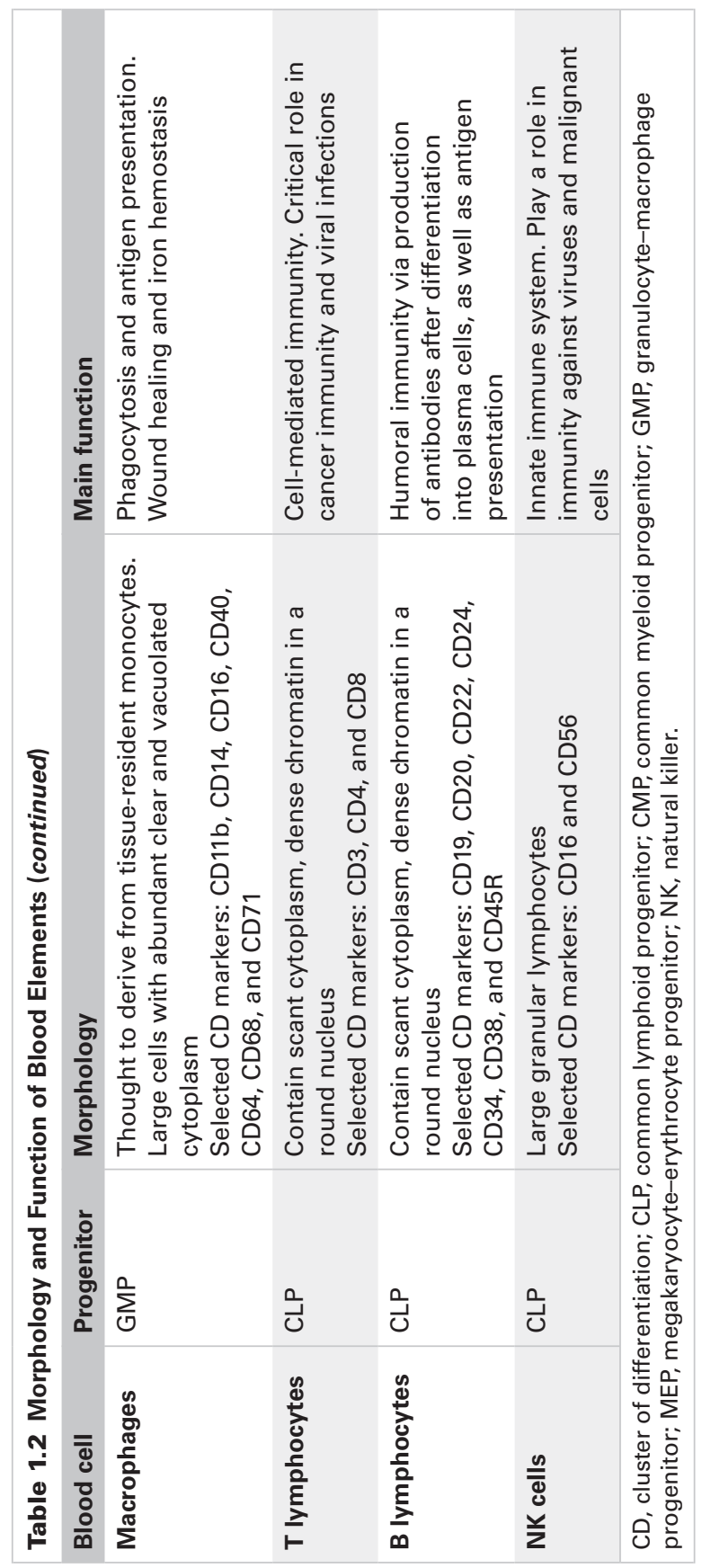


Table 1.3 Selected Clusters of Differentiation and Their Function

\begin{tabular}{|c|c|}
\hline $\begin{array}{l}\text { Cluster of } \\
\text { Differentiation }\end{array}$ & Function \\
\hline CD2 & Involved in T-cell adhesion \\
\hline CD4 & $\begin{array}{l}\text { Expressed on the surface of T helper cells. Acts } \\
\text { as a co-receptor for Class II MHC }\end{array}$ \\
\hline CD8 & $\begin{array}{l}\text { Expressed on the surface of T cytotoxic cells. } \\
\text { Acts as a co-receptor for Class I MHC }\end{array}$ \\
\hline CD11 & Promotes leukocyte adhesion \\
\hline CD 19, 20, and 21 & $\begin{array}{l}\text { B-cell surface antigens necessary for B-cell } \\
\text { signal transduction and function }\end{array}$ \\
\hline CD28 & $\begin{array}{l}\text { A co-stimulatory molecule present on the T-cell } \\
\text { surface necessary for its immune function }\end{array}$ \\
\hline CD34 & $\begin{array}{l}\text { Thought to promote cell adhesion and/or cell } \\
\text { cycle arrest. It is often used as a marker for } \\
\text { identifying and selecting hematopoietic stem } \\
\text { cells }\end{array}$ \\
\hline CD40 & $\begin{array}{l}\text { A ligand for B cells and involved in } \\
\text { immunoglobulin class switching }\end{array}$ \\
\hline $\begin{array}{l}\text { CD } 44,54,58, \text { and } \\
\text { CD62L }\end{array}$ & Function as adhesion molecules \\
\hline CD 80 and 86 & $\begin{array}{l}\text { Act as co-stimulatory receptors necessary for } \\
\text { the function of antigen-presenting cells }\end{array}$ \\
\hline CD90 & Enhances adhesion of T cells to stromal cells \\
\hline CD95 & Triggers apoptosis \\
\hline CD110 & $\begin{array}{l}\text { Receptor for thrombopoietin, the major } \\
\text { regulator for formation of megakaryocytes and } \\
\text { platelets }\end{array}$ \\
\hline $\begin{array}{l}\text { CD117 } \\
\text { (c-KIT receptor) }\end{array}$ & $\begin{array}{l}\text { Proliferation and survival of HSCs. It is often } \\
\text { used as a marker to identify HSCs }\end{array}$ \\
\hline CD150 & Lymphocyte proliferation \\
\hline CD154 & $\begin{array}{l}\text { B-cell receptor that promotes proliferation } \\
\text { and is also involved in immunoglobulin class } \\
\text { switching }\end{array}$ \\
\hline CD164 & $\begin{array}{l}\text { Involved in blood cell homing and suppressing } \\
\text { the proliferation of CD34+/CD38- cells }\end{array}$ \\
\hline
\end{tabular}




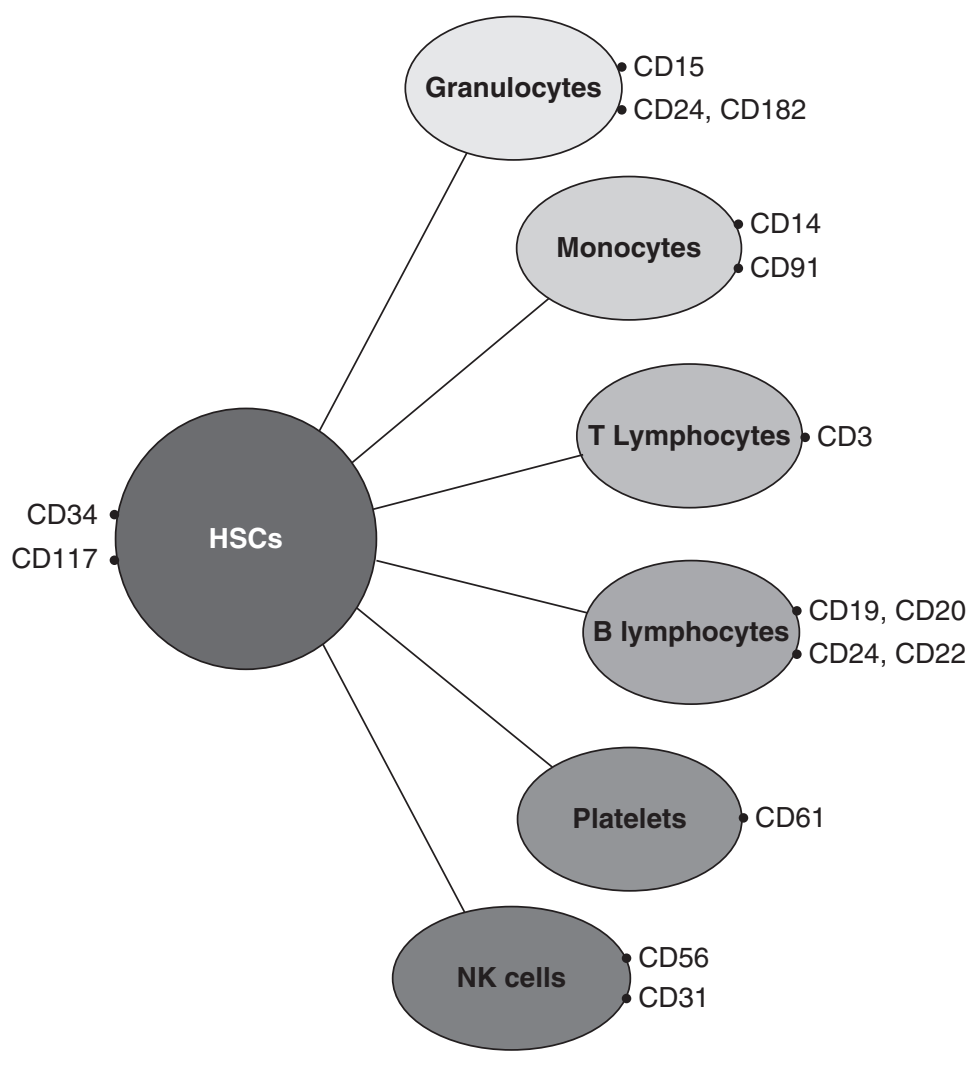

Figure 1.3 Selected CDs that belong to the different cell lineages.

$\mathrm{CD}$, cluster of differentiation; HSC, hematopoietic stem cell; NK, natural killer.

\section{BONE MARROW MICROENVIRONMENT}

\section{Bone Marrow Niches}

The bone marrow stroma comprises the non-hematopoietic components of bone marrow that provide various growth factors needed to sustain hematopoietic hemostasis and guide trafficking of hematopoietic cells. Many of these components are necessary for the HSCs and progenitors to proliferate and differentiate into various cell lineages. Among the key components are the bone marrow niches. A niche is defined as a local tissue microenvironment involved in maintaining and regulating a specific kind of stem or progenitor cell (12). Importantly, HSCs are thought to colonize two specific niches: the endosteal niche and the vascular niche. The 
endosteal niche is located between the endocortical surface and the trabecular surface of the bone (2). On the other hand, the vascular niche is located close to sinusoidal endothelial cells and bone marrow stromal cells (2). Current evidence indicates that these two niches, among other factors, play a key role in regulating the function of HSCs, including fate determination, although the relative importance of each of these niches is debated.

In the endosteal niche, a specific type of osteoblast is thought to play a role in HSC maturation, as it expresses $\mathrm{N}$-cadherin, which facilitates the interaction and binding to HSCs also expressing $\mathrm{N}$-cadherin (2). The role of $\mathrm{N}$-cadherin is, however, controversial, with some studies showing that it is not required for the maintenance of HSCs (13). Another protein, angiopoietin receptor-2 (Tie2), on the surface of HSCs, interacts with angiopoietin-1 (Ang-1) expressed on the surface of the lining osteoblasts in the endosteal niche (see Figure 1.4), which leads to enhanced survival of HSCs. In addition, other factors in the endosteal niche have been shown to influence survival and function of HSCs in animal studies (2). In the vascular niche, on the other hand, HSCs lie in close proximity to sinusoidal endothelial cells and stromal cells that seem to be critical for their maturation, with hematopoiesis being thought to take place in the extravascular area between the sinuses (2). Figure 1.4 illustrates both types of niches and the supporting cells involved. In mouse models, evidence suggests that fibroblast growth factor-1 (FGF-1) plays a role in mediating the crosstalk between the two niches to regulate hematopoiesis (14).

Homing is the process that enables circulating HSCs and progenitors to find their way to the bone marrow stroma and selectively reside there. This process is enabled by specific stromal cells and matrix proteins, and is crucial for the maintenance of longterm hematopoiesis. In addition, this property of HSCs is key to the success of stem cell transplantation. HSCs and progenitors interact with endothelial cells and the bone marrow microenvironment via selectins (e.g., cytoadhesion molecules $E, P$, and $L$ ), integrins (discussed in a separate section), and chemokines (e.g., CXCR4 binding to CXCL12) (7).

Drugs that inhibit the interaction of CXCR4 in HSCs and CXCL12 in stromal cells, such as plerixafor, can disrupt the homing steady state and allow mobilization of HSCs into peripheral blood. These drugs can be used to facilitate collection of HSCs from peripheral blood. Nonetheless, the first agent to be used specifically for this purpose was granulocyte colony-stimulating factor (G-CSF) or filgrastim. Apart from its major role in the differentiation and maturation of neutrophils, G-CSF leads to decreased production or increased degradation of stem cell retention factors, including CXCL12, Ang-1, Kit ligand, and vascular adhesion molecule-1 (VCAM-1) (7). The full 


\section{Osteoblast}

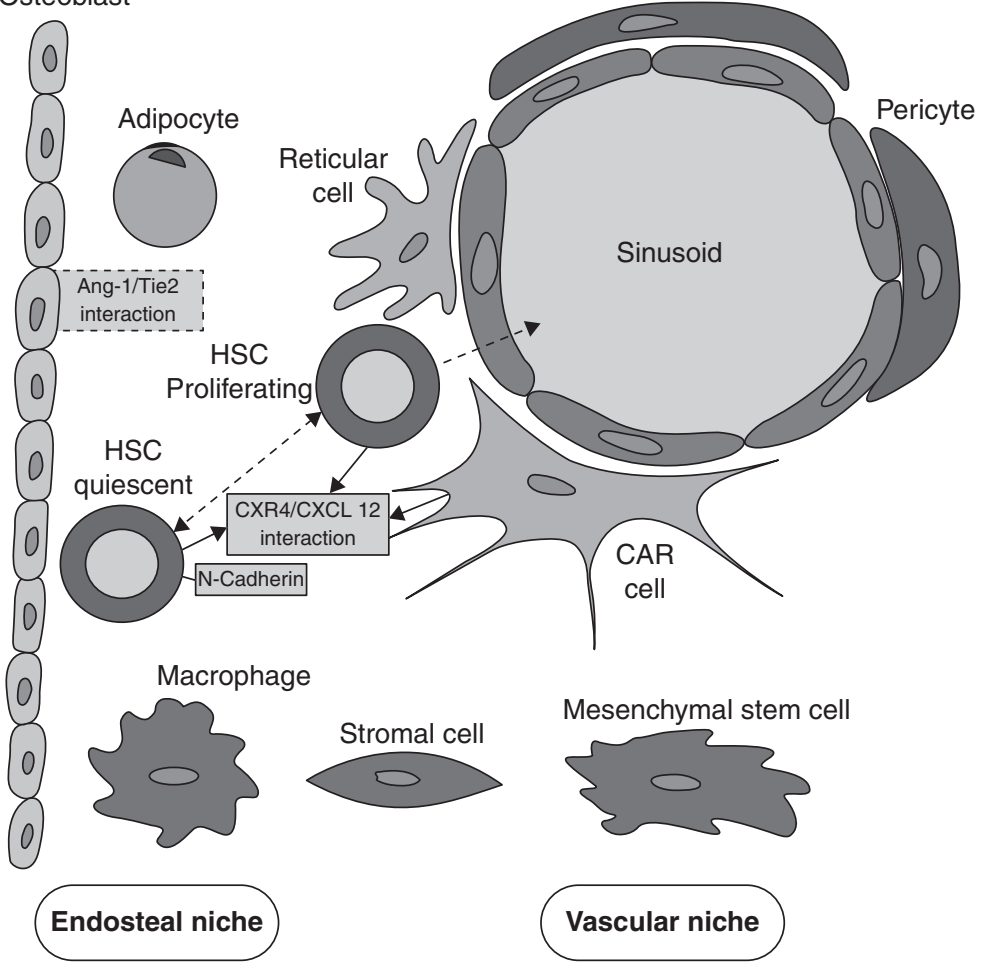

Figure 1.4 Illustration of both endosteal and vascular niches as well as the supporting cells involved.

Ang-1, angiopoietin-1; HSC, hematopoietic stem cell.

effects of G-CSF on HSCs are not entirely known, however. Recent animal studies show that G-CSF promotes HSCs' expansion and quiescence, while reducing their repopulating activity. This effect on hematopoietic cell activity occurs at least in part through activation ofToll-like receptor signaling (15). Furthermore, studies in mice indicate that bone marrow macrophages produce factors promoting osteoblast lineage maintenance and subsequently retention of stem cells. G-CSF inhibits the production of these factors, leading to stem cell mobilization (16). Notably, before filgrastim and plerixafor were available, HSC mobilization into peripheral blood was achieved with chemotherapeutic agents, such as cyclophosphamide, which kill rapidly dividing progenitors and thus trigger a burst of hematopoietic repopulation and HSC proliferation. 


\section{SUPPORTING CELLS}

The bone marrow microenvironment includes a multitude of supporting cells that provide the optimal soil for normal hematopoiesis. These include stromal cells, osteoblasts, osteocytes, osteoclasts, vascular endothelial cells, macrophages, and others (17). Several stromal cell types, such as reticular cells and mesenchymal stromal cells, have been described by different authors. Whether they represent completely distinct cellular entities or a spectrum of a single population is unclear. For instance, a population of reticular cells has long cytoplasmic processes that mesh together to form a lattice along the vascular sinuses, and evidence suggests that it has a role in sustaining HSCs in a proliferative and undifferentiated condition (18). This framework may be further enhanced by other stromal cells that support developing blood cells via cell-to-cell support (being an important element of niches) as well as by producing key hematopoietic growth factors. For example, mesenchymal stem cells (MSCs) can differentiate into osteolineage cells, chondrocytes, adipocytes, and mature stromal cells, all of which help support hematopoiesis. Mature T lymphocytes are also thought to provide adhesion molecules, growth factors, and cytokines that enhance the longevity, maturation, and differentiation of the hematopoietic hierarchy. Table 1.4 lists the different cells in the bone marrow microenvironment and their postulated role in maintaining normal hematopoiesis (19-21).

\section{Regulation and Control}

HSCs' self-renewal and lineage fate determination is a complex process controlled via various mechanisms working in harmony to ensure the HSC population is maintained while sustaining the production of different lineages. In addition, hematopoiesis has the capacity to ramp up production to meet excess demand in certain situations. This process is highly regulated but incompletely understood. In general, it is believed that there is a network of intercellular signals triggered by direct cell-to-cell interactions, cell-to-matrix interactions, or in response to cytokines and growth factors. Other factors involved include intracellular transcription factors, cell cycle regulators, and cell adhesion molecules, all subject to genetic as well as epigenetic regulation.

\section{Hematopoietic Growth Factors}

Growth factors are secreted molecules that influence cell growth and proliferation through interaction with target receptors on the cell surface and subsequent activation of intracellular signaling cascades and the effects thereof. Prominent hematopoietic growth 


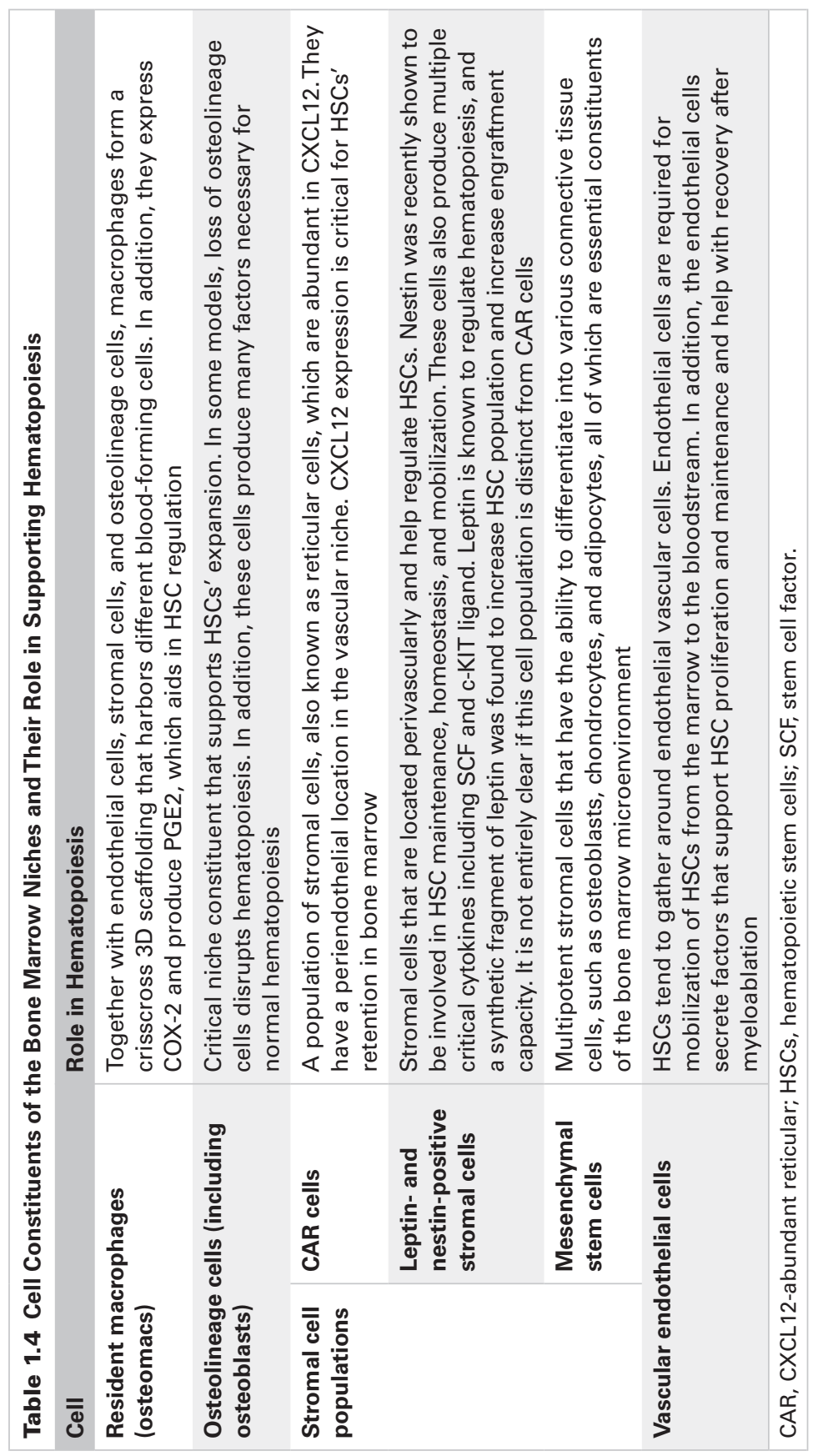


factors include stem cell factor (SCF, also known as C-KIT ligand), cytokines such as interleukin (IL)-3 and IL-6, erythropoietin (EPO), thrombopoietin (TPO), granulocyte-macrophage colony-stimulating factor (GM-CSF), G-CSF, and macrophage colony-stimulating factor (M-CSF). Importantly, some of these growth factors are made by the stromal cells in the bone marrow niches.

Regulatory factors may be loosely divided into those that affect the hematopoietic process as a whole (via modulation of early progenitors) and those that act exclusively on specific lineages. General factors that affect overall hematopoiesis especially during its early stages include SCF, IL-3, GM-CSF, IL-11, IL-6, and IL-1. On the other hand, lineage-specific growth factors, including EPO, TPO, G-CSF, M-CSF, and IL-5, are necessary for the production of red blood cells (RBCs), platelets, neutrophils, monocytes, and eosinophils, respectively. Table 1.5 and Figure 1.5 outline the main cytokines and growth factors that regulate the production and differentiation across the hematopoietic hierarchy (9). In addition, Figure 1.6 illustrates the main hematopoietic growth factors that act on specific progenitors.

While numerous growth factors affect hematopoiesis, some are thought to have a key role driving each of the major cell lines. For RBCs, the critical growth factor is EPO. EPO targets committed erythroid progenitors and leads to further differentiation and RBC production. It is also involved in keeping the RBC number within physiologic range via modulation of apoptosis of erythroid progenitors. EPO is synthesized and its levels regulated primarily via specialized cells in the renal cortex that sense blood oxygenation levels. Transcription of the EPO gene in these cells is regulated by hypoxia-inducible factor (HIF). EPO binds to EPO receptors on erythroid progenitors with subsequent signal transduction via the JAK2/STAT5 pathway (22) and promotion of erythropoiesis (7). Constitutive, unregulated JAK2 activation is one of the underlying genetic mechanisms for polycythemia vera, a disorder that manifests with excess RBC numbers (23). Interestingly, primitive erythrocytes need much less EPO to develop in contrast to definitive erythrocytes that arise later in life (24). Recombinant forms of EPO are used therapeutically in a number of diseases that are associated with primary anemia. Major indications include anemia related to renal failure, myelodysplastic syndrome (MDS), cancer-related anemia, and situations where patients refuse blood transfusions (e.g., due to certain beliefs). However, the use of recombinant EPO is governed by strict guidelines with the aim to limit the risk of side effects, namely, thromboembolic complications.

Development and production of platelets is chiefly regulated by TPO, largely produced by hepatocytes. TPO functions via activation 
Table 1.5 Selected Key Cytokines and Growth Factors Involved in Hematopoiesis

\begin{tabular}{|c|c|c|}
\hline Cytokine & Target & Effect \\
\hline IL-5 & $\begin{array}{l}\text { Eosinophils } \\
\text { B cells }\end{array}$ & $\begin{array}{l}\text { - Eosinophils: Differentiation, maturation, } \\
\text { and survival. It is also a mediator for } \\
\text { eosinophil activation } \\
\text { - B cells: Promotes B-cell growth and } \\
\text { immunoglobulin secretion }\end{array}$ \\
\hline IL-7 & $\begin{array}{l}\text { Early } \\
\text { lymphocytes }\end{array}$ & $\begin{array}{l}\text { - Growth regulator of lymphocytes. It is } \\
\text { secreted by the stromal cells. It is also } \\
\text { important for T-cell memory function, } \\
\text { survival, and homeostasis }\end{array}$ \\
\hline IL-2 & T cells & $\begin{array}{l}\text { - Important factor for the differentiation, } \\
\text { growth, and function of T cells. It was } \\
\text { initially known as T-cell growth factor. It } \\
\text { is important to maintain Tregs and for } \\
\text { the differentiation of CD4+T cells into } \\
\text { T-cell subsets following antigen presen- } \\
\text { tation and activation. Also promotes } \\
\text { differentiation into memory cells }\end{array}$ \\
\hline IL-4 & $\begin{array}{l}B \text { cells and } T \\
\text { cells }\end{array}$ & $\begin{array}{l}\text { B cells: Promotes the growth, helps in } \\
\text { activation, and modulates immunoglob- } \\
\text { ulin class switching. IL-4 is sometimes } \\
\text { referred to as B-cell growth factor } \\
\text { - T cells: Induces differentiation and } \\
\text { proliferation }\end{array}$ \\
\hline IL-15 & T cells, NK cells & $\begin{array}{l}\text { - T cells: Regulates T-cell activity and } \\
\text { homeostasis. It works with other factors } \\
\text { including IL-2 and IL-7 to maintain both } \\
\text { naïve and memoryT cells. It also en- } \\
\text { hances the survival of antigen-specific } \\
\text { CD8+T cells } \\
\text { - NK cells: Proliferation and activation } \\
\text { (through interacting with the compo- } \\
\text { nents of IL-2 receptor) }\end{array}$ \\
\hline SCF & $\begin{array}{l}\text { Primitive HSCs } \\
\text { and progenitors }\end{array}$ & $\begin{array}{l}\text { - General hematopoietic growth fac- } \\
\text { tor that acts to promote growth and } \\
\text { development of HSCs, progenitors, and } \\
\text { across all downstream lineages }\end{array}$ \\
\hline EPO & $\begin{array}{l}\text { Erythroid } \\
\text { progenitors }\end{array}$ & $\begin{array}{l}\text { Promotes survival and proliferation of } \\
\text { red blood cell progenitors as well as their } \\
\text { differentiation into mature red blood cells }\end{array}$ \\
\hline TPO & $\begin{array}{l}\text { Megakaryocyte } \\
\text { progenitors }\end{array}$ & $\begin{array}{l}\text { - Controls the proliferation and matura- } \\
\text { tion of megakaryocytic progenitors }\end{array}$ \\
\hline
\end{tabular}


Table 1.5 Selected Key Cytokines and Growth Factors Involved in Hematopoiesis (continued)

\begin{tabular}{|c|c|c|}
\hline Cytokine & Target & Effect \\
\hline M-CSF & $\begin{array}{l}\text { Monocyte } \\
\text { progenitors }\end{array}$ & $\begin{array}{l}\text { - Proliferation, differentiation, and surviv- } \\
\text { al of monocytic progenitors to sustain } \\
\text { macrophage homeostasis }\end{array}$ \\
\hline G-CSF & $\begin{array}{l}\text { Neutrophil } \\
\text { progenitors }\end{array}$ & $\begin{array}{l}\text { - A key stimulant of neutrophil produc- } \\
\text { tion. Has numerous clinical applications } \\
\text { such as stem cell mobilization for stem } \\
\text { cell transplantation and in treating } \\
\text { neutropenia }\end{array}$ \\
\hline \multicolumn{3}{|c|}{$\begin{array}{l}\text { EPO, erythropoietin; G-CSF, granulocyte colony-stimulating factor; } \\
\text { HSCs, hematopoietic stem cells; IL, interleukin; M-CSF, macrophage } \\
\text { colony-stimulating factor; NK, natural killer; SCF, stem cell factor;TPO, } \\
\text { thrombopoietin;Tregs, regulatory T cells. }\end{array}$} \\
\hline
\end{tabular}

of a cytokine receptor known as MPL present on bone marrow megakaryocytes and their precursors. The ultimate effect of TPO is to raise platelet numbers via initiation of signaling cascades leading to proliferation and differentiation of megakaryocytes from progenitor cells. TPO level is negatively regulated by platelet number, with increased platelets leading to downregulation of TPO mRNA (7). However, the mechanism is not fully understood. Although recombinantTPO has been used clinically to increase the platelet levels, its use was complicated by the development ofTPOneutralizing antibodies and consequent thrombocytopenia in several subjects (25). Thus, its use was abandoned. More recently, TPO receptor agonists (such as eltrombopag and romiplostim) have become available, and are not known to be associated with antibody formation. These drugs can be used to treat chronic immune thrombocytopenic purpura (ITP), aplastic anemia, and chronic hepatitis $\mathrm{C}$-associated thrombocytopenia.

The chief regulatory growth factors driving the granulocytic pathway are GM-CSF and G-CSF. Both GM-CSF and G-CSF activate specific receptors on myeloid precursors, with G-CSF acting on more mature progenitors, leading to subsequent activation of signaling pathways enhancing cell proliferation, survival, and activation of myeloid cells (26). G-CSF production is promoted by several factors including IL-1 $\beta$, tumor necrosis factor-alpha (TNF- $\alpha$ ), and lipopolysaccharide (LPS), all of which are usually elevated during infections or other stress. Therefore, G-CSF plays a critical role in mediating a granulopoietic stress response (15). Both recombinant GM-CSF (sargramostim) and G-CSF (filgrastim) are approved for the treatment of neutropenia (acquired and congenital) as well as 

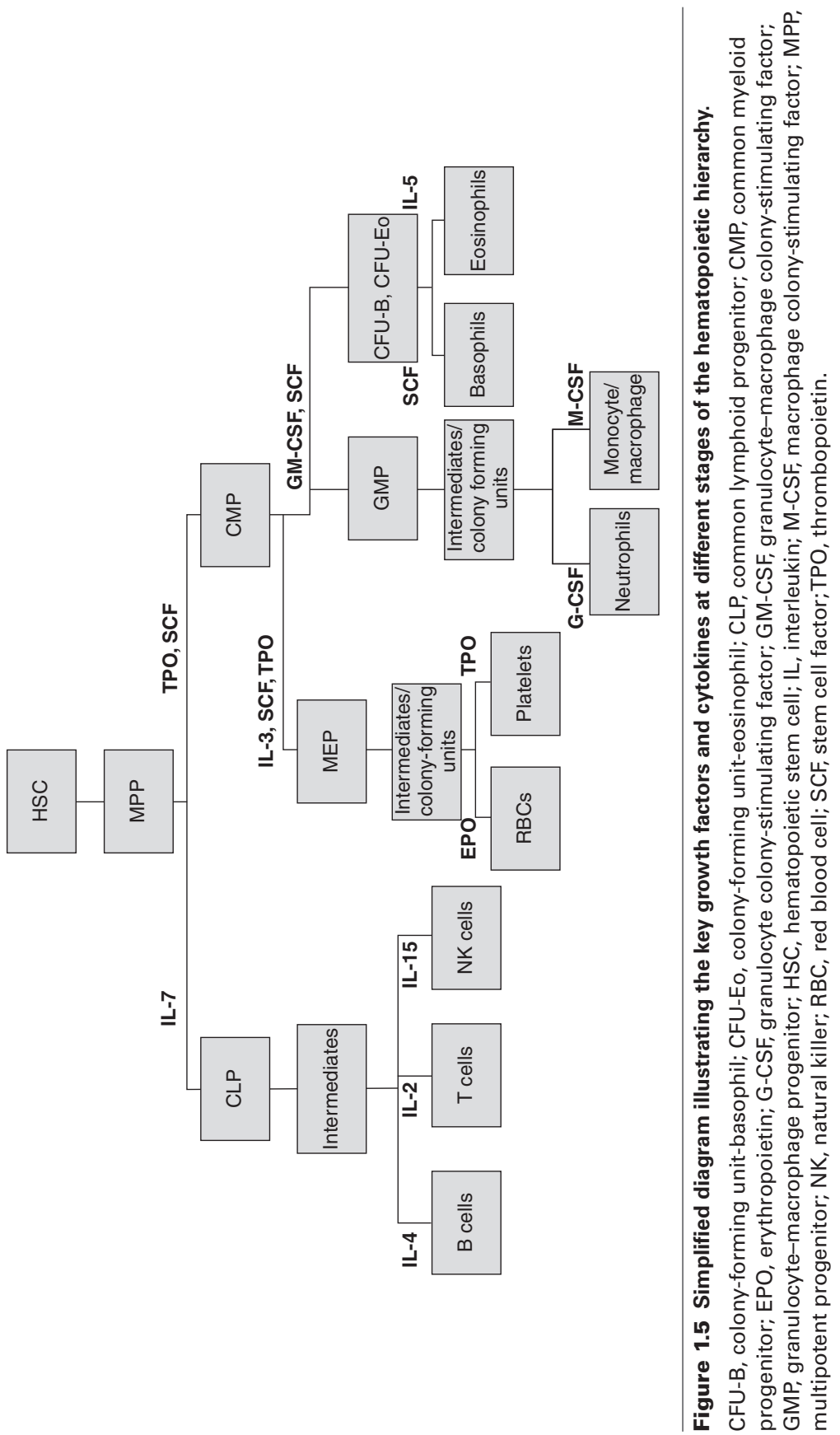


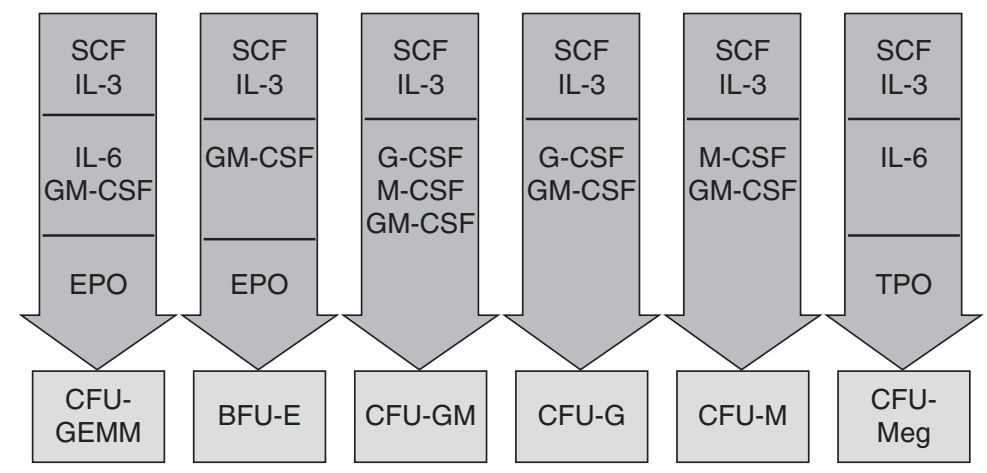

\section{Figure 1.6 Hematopoietic growth factors that target specific progenitors.}

BFU-E, burst-forming unit-erythroid; CFU-G, colony-forming unit-granulocyte; CFU-GEMM, colony-forming unit-granulocyte, erythrocyte, megakaryocyte, macrophage; CFU-GM, colony-forming unit-granulocyte monocyte; CFU-M, colony-forming unit-monocyte; CFU-Meg, colony-forming unit-megakaryocyte; EPO, erythropoietin; G-CSF, granulocyte colony-stimulating factor; GM-CSF, granulocyte-macrophage colony-stimulating factor; IL, interleukin; M-CSF, macrophage colony-stimulating factor; SCF, stem cell factor; TPO, thrombopoietin.

for mobilization of HSCs to peripheral blood prior to stem cell harvest. The mechanisms by which G-CSF mobilizes HSCs into peripheral blood are discussed under the section "Bone Marrow Niches." A pegylated form of G-CSF (pegfilgrastim) has the advantage of having sustained action (up to 2 weeks), eliminating the need for daily injections, and is also approved for treatment of chemotherapy-induced neutropenia. Currently, there are only limited data comparing G-CSF and GM-CSF and the results are conflicting.

\section{Integrins}

Integrins are transmembrane proteins that facilitate communication between the cell and its extracellular surrounding. They are composed of alpha and beta subunits, both having extracellular and intracellular domains. Ligand binding to the integrin surface domain leads to signal transduction with diverse outcomes, including cell cycle modulation among many other effects. Integrins also have an important role in homing of HSCs to bone marrow stroma and its microenvironment.

HSCs and primitive hematopoietic cells harbor many integrins. Among these is $\alpha_{4} \beta_{1}$ integrin, which binds to VCAM- 1 or fibronectin and promotes progenitor cell adhesion (27). Other integrins involved in hematopoiesis include $\alpha_{4} \beta_{7}$ and $\alpha_{5} \beta_{1}$ integrins (7). Of note, the avidity of progenitor cell integrins can be influenced by cytokines and growth factors including SCF, TPO, and CXL12. 
VCAM-1, fibronectin, as well as many other integrin ligands are highly expressed in the bone marrow microenvironment. Interactions of these molecules with integrins are thought to contribute to the retention of HSCs in the bone marrow (9).

\section{Intracellular Factors}

Transcription factors and other elements such as microRNAs and epigenetic regulators play a unique role in controlling gene transcription. These factors seem to define a road map to guide the hematopoietic process in terms of lineage and differentiation. Similar to growth factors, hematopoietic transcription factors may be divided into those that affect HSCs and early progenitors, and those that are lineage specific. Transcription factors have an important role in regulating HSC expansion as well as in guiding differentiation toward lymphoid and myeloid fates. Important examples are GATA-1, GATA-2, and PU.1. GATA-1 is critical for the differentiation of mature erythrocytes and megakaryocytes. GATA-2 seems to have a role in the final stages of megakaryocyte-erythrocyte progenitor (MEP) differentiation (9). PU.1, on the other hand, has an important role in the development of B cells and macrophages (7). Interestingly, lineage-specific transcription factors promote the expression of lineage-specific genes and work to counteract transcription factors that promote alternative lineages steering the hematopoietic machinery toward a certain lineage when needed (7). In addition, many lineage-specific transcription factors have a positive feedback effect and stimulate their own genes (autoregulation). Of relevance, transcription factors belonging to the HOX family are involved in influencing cell decisions including selfrenewal (9).

The specific transcription factors active in a particular cell ultimately influence its gene expression profile, which in turn guides the fate of HSCs and other hematopoietic cells. At each step in the hierarchy, the genes linked to the committed lineage either remain expressed or even become upregulated, while the genes related to the other lineages become silenced. This expression pattern was suggested by studies showing that primitive HSCs express the genes affiliated with multiple lineages, but downstream lineage-committed progenitors express only the genes specific to that lineage $(28,29)$. Mouse studies demonstrate that genes enriched in HSCs include developmental genes, some of which are associated with self-renewal. On the other hand, genes enriched in lineage-committed progenitor cells are linked to differentiation and regulation of immune responses. As HSCs develop into committed progenitors, there is downregulation of "stemness" genes and upregulation of lineage-specific genes (30). 
Apart from transcription factors, more recently recognized mechanisms of translational control have also been shown to play an important role in the overall hematopoietic process. MicroRNAs are small, noncoding RNAs that regulate the gene expression by silencing and degrading mRNA, with subsequent inefficient translation. In hematopoiesis, they function to regulate responses to cytokines and transcription factors, and thus have a key role in guiding differentiation and progenitor lineage commitment. Examples include miR-181, which has a role in B-cell differentiation (at least in animal models); miR-155, which inhibits erythropoiesis and megakaryopoiesis; and miR-520h, which is upregulated in primitive stem cells, suggesting a role in stem cell maintenance (9).

\section{KEY POINTS}

- Hematopoiesis is a highly regulated process with the end products being 10 formed elements: erythrocytes, platelets, neutrophils, monocytes, basophils, eosinophils, B lymphocytes, $T$ lymphocytes, NK cells, and dendritic cells.

- Sites of hematopoiesis change during development.

- In the embryo, hematopoiesis occurs in the yolk sac. In the fetus, it occurs in the liver, spleen, placenta, and bone marrow. After birth, hematopoiesis occurs solely in the bone marrow, except in states of inefficient hematopoiesis. In such states, hematopoiesis may revert to its fetal sites (liver and spleen).

- Self-renewal and differentiation are the key characteristics of HSCs, which are essential for hematopoiesis.

- The main steps of hematopoiesis include self-renewal of HSCs, HSCs' differentiation, commitment to specific lineages, and finally, maturation and development of lineage-committed progenitors into terminally mature cells.

- As HSCs differentiate into different lineages, their CD expression changes. CDs are thus used to identify the different cells in the hierarchy and have numerous clinical and research applications.

- The bone marrow microenvironment is essential for normal hematopoiesis. The endosteal and vascular niches are thought to play an important role in its preservation and regulation.

- Regulatory factors of hematopoiesis can be classified as external growth factors (such as EPO, TPO, and G-CSF), integrins, and intracellular factors (such as transcription factors, microRNAs, and others).

- Many of the external regulatory factors are produced by the supporting cells in the bone marrow niches. 


\section{REFERENCES}

1. Aqmasheh $S$, Shamsasanjan $K$, Akbarzadehlaleh $P$, et al. Effects of mesenchymal stem cell derivatives on hematopoiesis and hematopoietic stem cells. Adv Pharm Bull. 2017;7(2):165-177. doi:10.15171/apb.2017.021

2. Tamma R, Ribatti D. Bone niches, hematopoietic stem cells, and vessel formation. Int J Mol Sci. 2017;18(1):151. doi:10.3390/ ijms18010151

3. Golub R, Cumano A. Embryonic hematopoiesis. Blood Cells Mol Dis. 2013;51(4):226-231. doi:10.1016/j.bcmd.2013.08.004

4. ChenT, Wang F, Wu M, Wang ZZ. Development of hematopoietic stem and progenitor cells from human pluripotent stem cells. J Cell Biochem. 2015;116(7):1179-1189. doi:10.1002/jcb.25097

5. Tavian M, Peault B. Embryonic development of the human hematopoietic system. Int J Dev Biol. 2005;49(2-3):243-250. doi:10.1387/ijdb.041957mt

6. Wagers AJ, Christensen JL, Weissman LL. Cell fate determination from stem cells. Gene Ther. 2002;9(10):606-612. doi:10.1038/sj.gt.3301717

7. Greer JP, Arber DA, Glader, B, et al. Wintrobe's Clinical Hematology. 13th ed. Philadelphia, PA: Wolters Kluwer Health | Lippincott Williams \& Wilkins; 2014.

8. Frisch BJ, Calvi LM. Hematopoietic stem cell cultures and assays. Methods Mol Biol. 2014;1130:315-324. doi:10.1007/978 -1-62703-989-5_24

9. Kaushansky K. Hematopoietic stem cells, progenitors, and cytokines. In: Kaushansky K, Lichtman M, Beutler E, et al., eds. Williams Hematology. NewYork, NY: McGraw-Hill Education; 2015.

10. Terstappen LW, Huang S, Safford M, et al. Sequential generations of hematopoietic colonies derived from single nonlineage-committed CD34+CD38- progenitor cells. Blood. 1991;77(6):1218-1227.

11. Takahashi M, Matsuoka Y, Sumide K, et al. CD133 is a positive marker for a distinct class of primitive human cord bloodderived CD34-negative hematopoietic stem cells. Leukemia. 2014;28(6):1308-1315. doi:10.1038/leu.2013.326

12. Morrison SJ, Scadden DT.The bone marrow niche for haematopoietic stem cells. Nature. 2014;505(7483):327-334. doi:10.1038/ nature12984

13. Greenbaum AM, Revollo LD, Woloszynek JR, et al. N-cadherin in osteolineage cells is not required for maintenance of hematopoietic stem cells. Blood. 2012;120(2):295-302. doi:10.1182/ blood-2011-09-377457 
14. de Haan $G$, Weersing $E$, Dontje B, et al. In vitro generation of long-term repopulating hematopoietic stem cells by fibroblast growth factor-1. Dev Cell. 2003;4(2):241-251. doi:10.1016/ S1534-5807(03)00018-2

15. Schuettpelz LG, Borgerding JN, Christopher MJ, et al. G-CSF regulates hematopoietic stem cell activity, in part, through activation of Toll-like receptor signaling. Leukemia. 2014;28(9): 1851-1860. doi:10.1038/leu.2014.68

16. Christopher MJ, Rao M, Liu F, et al. Expression of the G-CSF receptor in monocytic cells is sufficient to mediate hematopoietic progenitor mobilization by G-CSF in mice. J Exp Med. 2011;208(2):251-260. doi:10.1084/jem.20101700

17. Anthony BA, Link DC. Regulation of hematopoietic stem cells by bone marrow stromal cells. Trends Immunol. 2014;35(1):3237. doi:10.1016/j.it.2013.10.002

18. Nagasawa T, Omatsu $Y$, Sugiyama T. Control of hematopoietic stem cells by the bone marrow stromal niche: the role of reticular cells. Trends Immunol. 2011;32(7):315-320. doi:10.1016/ j.it.2011.03.009

19. Calvi LM, Link DC. Cellular complexity of the bone marrow hematopoietic stem cell niche. Calcif Tissue Int. 2014;94(1): 112-124. doi:10.1007/s00223-013-9805-8

20. Xie L, Zeng X, Hu J, Chen Q. Characterization of Nestin, a selective marker for bone marrow derived mesenchymal stem cells. Stem Cells Int. 2015;2015:762098. doi:10.1155/2015/ 762098

21. Dias CC, Nogueira-Pedro A, Tokuyama PY, et al. A synthetic fragment of leptin increase hematopoietic stem cell population and improve its engraftment ability. J Cell Biochem. 2015;116(7):1334-1340. doi:10.1002/jcb.25090

22. Kuhrt D, Wojchowski DM. Emerging EPO and EPO receptor regulators and signal transducers. Blood. 2015;125(23):3536-3541. doi:10.1182/blood-2014-11-575357

23. Jamieson CHM, Gotlib J, Durocher JA, et al. The JAK2 V617F mutation occurs in hematopoietic stem cells in polycythemia vera and predisposes toward erythroid differentiation. Proc Natl Acad Sci. 2006;103(16):6224-6229. doi:10.1073/pnas.0601462103

24. Palis J,Yoder MC. Yolk-sac hematopoiesis: the first blood cells of mouse and man. Exp Hematol. 2001;29(8):927-936. doi:10.1016/ S0301-472X(01)00669-5

25. Li J, Yang C, Xia Y, et al. Thrombocytopenia caused by the development of antibodies to thrombopoietin. Blood. 2001;98(12):3241-3248. doi:10.1182/blood.V98.12.3241 
26. Panopoulos AD, Watowich SS. Granulocyte colony-stimulating factor: molecular mechanisms of action during steady state and 'emergency' hematopoiesis. Cytokine. 2008;42(3):277-288. doi:10.1016/j.cyto.2008.03.002

27. Vanderslice P, Biediger RJ, Woodside DG, et al. Small molecule agonist of very late antigen-4 (VLA-4) integrin induces progenitor cell adhesion. J Biol Chem. 2013;288(27):19414-19428. doi:10.1074/jbc.M113.479634

28. Miyamoto T, Iwasaki $\mathrm{H}$, Reizis $\mathrm{B}$, et al. Myeloid or lymphoid promiscuity as a critical step in hematopoietic lineage commitment. Dev Cell. 2002;3(1):137-147. doi:10.1016/S1534-5807(02)00201-0

29. Kiel MJ, Yilmaz OH, IwashitaT, et al. SLAM family receptors distinguish hematopoietic stem and progenitor cells and reveal endothelial niches for stem cells. Cell. 2005;121(7):1109-1121. doi:10.1016/j.cell.2005.05.026

30. Terskikh AV, MiyamotoT, Chang $C$, et al. Gene expression analysis of purified hematopoietic stem cells and committed progenitors. Blood. 2003;102(1):94-101. doi:10.1182/blood-2002-08-2509 